\title{
El trabajo de las personas con discapacidad ante la crisis
}

\author{
Vanesa Rodríguez \\ Departamento de Sociología. Universidad de Oviedo \\ rodriguezvanesa@uniovi.es \\ Begoña Cueto \\ Departamento de Economía Aplicada. Universidad de Oviedo \\ bcueto@uniovi.es
}

Resumen: En un contexto de crisis económica como el actual, determinados colectivos, que ya partían de una peor posición dentro del mercado de trabajo, se han visto más afectados por la destrucción de empleo. El objetivo con el que se plantea este trabajo es identificar cómo ba afectado el cambio de ciclo económico al empleo de las personas con discapacidad, distinguiendo entre aquellos que prestan servicios en un centro especial de empleo (CEE) y los que lo hacen en la empresa ordinaria.

Para ello se utilizan datos de la Muestra continua de vidas laborales (MCVL) en sus oleadas de 2005 a 2011, lo que nos permite realizar un seguimiento longitudinal de los individuos en dos momentos de muy distinta coyuntura económica: bonanza $y$ crisis. A través de la MCVL podemos identificar a trabajadores con discapacidad tanto en empresas ordinarias como en CEE, lo que nos permite estudiar qué tipo de centro ofrece una mejor "protección» al empleo de sus trabajadores con discapacidad frente a la crisis. Los resultados preliminares muestran que son los trabajadores con una mayor relación con los CEE los que ven reducida en menor proporción su participación en el empleo.

Palabras clave: discapacidad, estabilidad, empleo protegido, empleo ordinario. 
The employment of people with disabilities in times of economic retrenchment Abstract: Certain groups of workers have been much more affected by job losses as a result of the current economic crisis. The aim of this paper is to identify how the economic cycle has affected the employment of people with disabilities both in sheltered employment centers (SECs) and in ordinary firms. We analyze data from the 2005 2011 Longitudinal Sample of Working Lives to determine the employment stability of disabled people during contrasting economic climates of prosperity and crisis. Our results show that disabled workers in SECs have greater stability than disabled workers in ordinary firms. In addition, the results indicate that SECs act as an «employment refuge» for disabled people in times of crisis. However, workers in SECs find it more difficult to find a job outside these organizations.

Keywords: disability, stability, sheltered employment, ordinary employment. 


\section{Introducción}

Las personas con discapacidad presentan una menor participación en el mercado laboral y menores tasas de empleo. Además, el empleo ordinario no es una opción viable para algunas personas con discapacidad, por lo que el empleo protegido es una de las medidas adoptadas para promover las oportunidades de empleo para este colectivo.

Sin embargo, las diferencias en el diseño de este tipo de programas entre distintos países (e incluso dentro de los países) pueden ser significativas. En algunos casos, el empleo protegido puede aislar a los trabajadores con discapacidad $y$ ofrecer bajos salarios y unas relaciones laborales precarias. Sin embargo, otras formas de empleo protegido son similares al trabajo productivo en las empresas ordinarias. En varios países, durante las últimas décadas, este tipo de empleo ha perdido su papel preponderante como empleo protegido para las personas con discapacidad, debido a la implementación de otras medidas como el empleo con apoyo. Los países con mayor porcentaje de empleo protegido son Bélgica, Italia y España (Shima et aliii, 2008).

En España, la Ley de Integración Social del Minusválido (LISMI), aprobada en 1982, establece que el empleo ordinario de las personas con discapacidad debe ser un objetivo primordial de las políticas de empleo. Esta norma considera los centros especiales de empleo (CEE) — principal vía de integración en empleo de las personas con discapacidad mediante la fórmula del trabajo protegido- como el medio de emplear a aquellas personas con discapacidad que, por razón de la naturaleza o de las consecuencias de sus discapacidades no pudieran, provisional o definitivamente, ejercer una actividad laboral en unas condiciones normales. En resumen, la intención de esta norma es que los CEE sirvan de puente entre el trabajo protegido y el ordinario.

En la actualidad y desde hace ya más de dos décadas, los CEE son uno de los pilares clave de las medidas dirigidas a la integración sociolaboral de las personas con discapacidad. Su actuación no ha estado exenta de polémica, con opiniones tanto a favor como en contra. Los argumentos a favor se centran en que los CEE contribuyen a reducir la inactividad de las personas con discapacidad, además de proporcionales un trabajo remunerado, favoreciendo su desarrollo personal. Los argumentos en contra señalan que los CEE, en lugar de integrar, pueden contribuir a la segregación de sus trabajadores, en la medida en que constituyen un punto de destino y no un medio de tránsito al empleo ordinario. Mientras que en los países de nuestro entorno se ha dado un paso más hacia el empleo con apoyo, en España los CEE son la medida de fomento del empleo de las personas con discapacidad que mayor volumen de recursos supone. 
Desde distintas organizaciones relacionadas con la discapacidad, así como desde ámbitos académicos y políticos, se argumenta que la función principal de estos centros es proporcionar trabajo $\mathrm{u}$ oportunidades profesionales a largo plazo para las personas con discapacidad, sin tener en cuenta si los trabajadores transitan hacia la empresa ordinaria. Por el contrario, otros expertos sostienen que los CEE deben ser un paso intermedio hacia un trabajo en una empresa ordinaria. Desde esta perspectiva, los trabajadores contratados por los CEE finalmente deben pasar a un trabajo en una empresa no protegida. Sin embargo, el debate sigue abierto porque hay muy pocos datos para apoyar claramente alguna de las visiones.

Ante esta perspectiva de los CEE como un mercado protegido para los trabajadores con discapacidad, nuestro objetivo es analizar la estabilidad del empleo de las personas con discapacidad en CEE durante el periodo 2005-2011, en comparación con el empleo en empresas ordinarias. Para ello, en el siguiente apartado se lleva a cabo una revisión de la literatura sobre el empleo de las personas con discapacidad, con una especial mención al empleo protegido. En el segundo apartado se indican las principales características de la base de datos utilizada en este análisis. A continuación, se presenta el análisis descriptivo de la muestra seleccionada y, por último, se presenta un breve apartado de conclusiones.

\section{Las políticas de integración en el mercado de trabajo dirigidas a personas con discapacidad}

A pesar de que en la actualidad se ve la integración laboral de las personas con discapacidad como una vía para conseguir el desarrollo pleno de su personalidad, a través de la independencia personal y económica que otorga la participación activa en el mercado de trabajo, esto no siempre ha sido así. En los últimos años se ha pasado de una política sobre discapacidad centrada en la garantía de ingresos a una política que ve el empleo como la principal herramienta de integración social de este colectivo.

Este cambio en la percepción de la importancia del empleo para las personas con discapacidad se aprecia también en la literatura académica; los estudios sobre discapacidad y mercado de trabajo son relativamente recientes, especialmente en el caso español. En la década de 1970 comienzan a aparecer estudios empíricos sobre la participación laboral de las personas con discapacidad en Estados Unidos; a partir de la década de los 90 se empiezan a llevar a cabo estudios de este tipo en el ámbito europeo, y a comienzos de este siglo aparecen los primeros 
estudios realizados en España (Baldwin y Schumacher, 2002; Gannon y Nolan, 2004; Malo, 2001, 2003 y 2004; Dávila, 2006).

En cuanto a los estudios centrados en el empleo protegido, aunque los CEE son un instrumento muy utilizado para intentar alcanzar la inserción laboral del colectivo de personas con discapacidad, son pocos los análisis, tanto nacionales como internacionales, que se han ocupado de aportar alguna evidencia empírica sobre el tema. En el caso español sí existe información sobre CEE, pero no está organizada con criterios estadísticos que permitan su análisis. Además tampoco tiene representatividad suficiente para el conjunto del país. En el ámbito internacional, son varios los autores que han revisado las formas que el empleo protegido toma en los distintos países (Thorton y Lunt, 1997; Delsen, 1996 y2001), pero pocos aportan datos sobre su uso y efectos en términos de inserción laboral de las personas con discapacidad. ${ }^{1}$

\subsection{Las personas con discapacidad y su relación con el mercado de trabajo}

Los estudios sobre la participación laboral de las personas con discapacidad coinciden en que tener alguna discapacidad afecta negativamente a la participación laboral, tanto en la tasa de paro como en la de actividad, y en que estas diferencias en participación con las personas sin discapacidad no se pueden explicar completamente por diferencias en características; es decir, hay una parte debida a discriminación hacia las personas con discapacidad o a diferencias de productividad no observadas relacionadas con la discapacidad.

Existe consenso en la literatura sobre que la participación laboral no es homogénea dentro del grupo de personas con discapacidad. Variables como el tipo de discapacidad, el género o la inversión en capital humano influyen en la probabilidad de participar en el mercado de trabajo, ya sea de forma negativa o positiva (Zwinkels, 2001; Dávila, 2003; Villa, 2003; Dávila y Malo, 2006; Malo et alii, 2009). Respecto al nivel educativo de las personas con discapacidad, Dávila (2003) constata la existencia de una brecha entre los niveles educativos de la población joven con discapacidad y la población joven en general. Giménez y Ramos (2003) estudian la discriminación de las mujeres con discapacidad en España, y concluyen que este colectivo hace un mayor uso de las pensiones no contributivas, mientras que accede en menor medida a las contributivas como consecuencia del desigual acceso al mercado de trabajo.

La investigación sobre el caso español muestra con claridad que el principal problema de las personas con discapacidad es su baja participación laboral.

1 Para un análisis más exhaustivo de la bibliografía existente consultar Cueto et aliii (2008). 
Así, Malo (2001, 2003, 2004), Dávila (2006), Malo et alii (2009) y Cueto et alii (2012) muestran que la tasa de actividad de las personas con discapacidad alcanza valores cercanos a la mitad de la tasa de actividad de las personas sin discapacidad.

Además, Cueto et alii (2012), al comparar los resultados obtenidos con la EDAD-2008 con los de la EDDES-1999, concluyen que las tasas de actividad y de empleo de las personas con discapacidad son la mitad de altas que las de las personas sin discapacidad, mientras que la tasa de paro es del doble, situación que apenas ha variado respecto a la registrada hace una década. Entre ambas encuestas las tasas de actividad y ocupación de las personas sin discapacidad han crecido de forma más notable que las de las personas con discapacidad.

La relación entre discapacidad y trabajo a tiempo parcial se menciona en la mayoría de los estudios sobre la participación laboral de este colectivo. En un exhaustivo análisis, Pagán (2012) concluye que las personas con discapacidad (especialmente las mujeres) tienen una mayor probabilidad de permanecer en trabajos a tiempo parcial que los trabajadores sin discapacidad.

Una vez que las personas con discapacidad se incorporan al mercado de trabajo, su ocupación se centra en trabajos a tiempo parcial y peor remunerados que los de los trabajadores sin discapacidad. Además, es más probable que sufran desempleo o inactividad (Zwinkels, 2001; Pascual y Cantarero, 2007).

Respecto al análisis de la satisfacción laboral, Pagán y Malo (2009) concluyen que esta es superior en el caso de las personas con discapacidad; la diferencia en satisfacción laboral parece obedecer a que las expectativas sobre el empleo de las personas con discapacidad son más bajas que las de las personas sin discapacidad. Resultados similares obtiene Pagán (2011a) al comparar la satisfacción laboral de los trabajadores de entre 50 y 64 años con y sin discapacidad en Europa.

El autoempleo de las personas con discapacidad también ha sido objeto de análisis por parte de la literatura. Pagán (2011b) encuentra que los trabajadores autónomos están más satisfechos con sus puestos de trabajo que los asalariados; sin embargo, no encuentra evidencia de que la relación entre autoempleo y satisfacción en el trabajo difiera entre personas mayores con discapacidad y sin discapacidad.

Los menores salarios de las personas con discapacidad son un tema recurrente en la literatura, aunque muchos trabajos no entran a evaluar a qué se deben estas diferencias salariales (Jones et alii, 2006; Humer et alii, 2007; Brown y Emery, 2008). Los que sí lo hacen observan que una parte de ella no se puede explicar por diferencias en características (Johnson y Lambrinos, 1985; Baldwin y Johnson, 2000; Pagán y Marchante, 2004; Malo y Pagán, 2012). Aunque 
tradicionalmente se relaciona el componente rendimientos - es decir, la parte de la diferencia salarial que no se puede explicar por las diferentes características de las personas con discapacidad - con la discriminación, en el caso de las personas con discapacidad esta diferencia se debe interpretar con precaución debido a que sus características pueden limitar realmente su productividad (Baldwin y Johnson, 1995; Malo y Pagán, 2012).

\subsection{El empleo protegido en la literatura}

Desde la década de los ochenta, el empleo protegido ha constituido la principal política de empleo dirigida a personas con discapacidad en la mayor parte de los países europeos. El planteamiento de esta medida se basa en que las personas con discapacidad no pueden desarrollar un empleo en un entorno ordinario y, por tanto, es necesaria la creación de empresas específicas para que este colectivo pueda insertarse en el mercado ordinario de trabajo.

La Comisión Europea (2000) ha elaborado un análisis de los sistemas de empleo protegido, mostrando sus características para cada país, y ha detectado que las tendencias de las políticas de empleo para las personas con discapacidad combinan elementos como la integración en el mercado de trabajo y las medidas para atajar la discriminación y reducir la dependencia de los subsidios. En los últimos años, ha aumentado la integración de las personas con discapacidad en el empleo ordinario, estimulada por medidas que buscan la igualdad de oportunidades. La aprobación de las directrices europeas de empleo ha incrementado la atención dada al paso de políticas de empleo pasivas a activas; es decir, del sostenimiento de rentas a la preparación y facilitación del paso al empleo. Esto ha llevado, en ocasiones, a las organizaciones de personas con discapacidad a interpretar que la razón que lleva a este cambio de políticas es la necesidad de ahorrar recursos, y no la conveniencia de fomentar el paso al mercado de trabajo ordinario. La descoordinación en las políticas o el miedo a perder ingresos también dificultan la puesta en marcha de medidas de activación.

En OCDE (2003) se subraya que, aunque el empleo protegido se considera inapropiado para grupos numerosos de personas con discapacidad y muchos países preferirían sustituirlo con iniciativas del tipo del empleo con apoyo, empíricamente no se han encontrado descensos significativos de este tipo de empleo por el momento. ${ }^{2}$ Whiteford (2007), en un artículo posterior, remarca nuevamente esta idea.

$2 \mathrm{Si}$ bien la OIT indica que las políticas para promover la igualdad de oportunidades en el empleo de personas con discapacidad deben incluir distintos tipos de empleo protegido para las personas con discapacidad a los que la entrada en el empleo ordinario no sea posible (Recomendación n. ${ }^{\circ} 168$ OIT —citado en O’Reilly, 2007). 
En el caso de España, la OCDE ha recomendado realizar un cambio en el gasto de políticas activas, que consistiría en reducir las ayudas al empleo protegido y usar ese dinero para una mejora de las capacidades y la empleabilidad de las personas con discapacidad, independientemente de que tengan certificado de discapacidad o no (OCDE, 2007). Las razones para esta recomendación se basan en los débiles resultados de este tipo de empleo. Sin embargo, se debe tener en cuenta que la legislación española establece la necesidad de estar en posesión del certificado de discapacidad igual o superior al 33\% o de la declaración de incapacidad permanente para tener la consideración de persona con discapacidad, lo que dificulta la implementación de esta recomendación.

En un estudio previo (OCDE, 1992), se analizó la experiencia en varios países concluyendo que la segregación inherente al empleo protegido hace que las personas con discapacidad no puedan participar de forma normal en la comunidad; la razón es que el trabajo en CEE se desarrolla en condiciones diferentes a las de las empresas ordinarias (sobreprotección y menor exigencia), de manera que los trabajadores con discapacidad no adquieren las capacidades necesarias para desenvolverse en el mercado de trabajo normal. También indica que en la mayor parte de países los salarios son bajos, hay una pobre protección laboral y el estado del trabajador es incierto. Por último, sitúa las tasas de transición entre el 1\% y el 5\%. Taylor (2003) y Gill (2005) coinciden en los bajos salarios de los trabajadores en CEE y en que la transición hacia el mercado de trabajo ordinario es un mito. Taylor (2003) cuantifica esta transición en un 3,5\%; además señala la baja incidencia de las discapacidades graves entre los trabajadores de CEE.

López y Seco (2005) señalan, aunque sin aportar datos al respecto, que los CEE en España se han convertido en un punto final para los trabajadores con discapacidad, principalmente para los que tienen problemas psíquicos leves, que o bien no encuentran la manera de pasar al empleo ordinario o bien temen perder la protección y estabilidad que tienen en el empleo protegido. Los autores hacen referencia a una posible precarización laboral e inestabilidad del colectivo como consecuencia del comportamiento oportunista de algunos empresarios.

Pereda et alii (2003) critican las pocas veces que se produce el objetivo de tránsito a la empresa ordinaria de los CEE (aunque tampoco ofrecen cifras al respecto). Esto produce un «efecto de campana de cristal» que proporciona a sus trabajadores una sensación de mayor seguridad, pero en un escenario de sobreprotección que limita sus expectativas y posibilidades de promoción laboral autónoma.

El estudio de Ibáñez y Mudarra (2005) incide en el perfil y en las relaciones sociales de personas con discapacidad dentro del empleo protegido en España, y 
concluye que los trabajadores con discapacidad son conscientes de sus dificultades para encontrar un empleo adecuado y, por ello, simplemente quieren trabajar.

Casado (2004) analiza el empleo de las personas con discapacidad. A partir de datos del Instituto Nacional de Estadística, estudia la presencia de personas con discapacidad en el mercado de trabajo, para posteriormente hacer un análisis de las distintas medidas creadas por la LISMI para el fomento del empleo, entre ellas los CEE. Destaca su labor para reducir el paro y la inactividad de este colectivo. También indica, como desventaja, que no respetan los principios de normalización e integración, pues en algunos casos existen espacios físicos separados de los trabajadores ordinarios. El autor, citando a Albor (1997), señala que los CEE son instrumentos de integración en el empleo mediante un régimen de segregación laboral, integradores y marginadores a la vez.

A partir de datos de contratación del Servicio Público de Empleo, Rodríguez (2012b) encuentra que, en los últimos seis años, ha aumentado el número de contratos realizados con personas con discapacidad como consecuencia del fuerte aumento de la contratación en CEE, en detrimento de las contrataciones en empresas ordinarias; los contratos celebrados por CEE casi se han duplicado desde 2006 y el incremento en la contratación se ha dado en los contratos temporales. La autora concluye que los CEE pueden estar desempeñando una función de «refugio de empleo» para las personas con discapacidad, evitando que estas pierdan el contacto con el mercado de trabajo durante la actual crisis. Esto es una ventaja para los trabajadores con discapacidad en CEE debido a que la actividad de estas empresas parece resultar menos afectada por la recesión, por lo que tienen mejores oportunidades para mantenerse en el mercado laboral.

Por otra parte, Rubio (2003) indica una serie de aspectos positivos de los CEE; proporcionan experiencia laboral, formación y capacitación profesional, y las personas con discapacidad adquieren habilidades y hábitos laborales y cierta polivalencia y flexibilidad (características valoradas en el mercado de trabajo ordinario). El autor también enumera una serie de motivos por los que los CEE no cumplen el objetivo normativo ni práctico con el que fueron creados, citando que no son centros de tránsito, ${ }^{3}$ que su objetivo es la productividad y competitividad en condiciones laborales precarias y sin sindicación, que no se aplican los convenios colectivos del sector o rama al que pertenecen y que no existe representación sindical. Por último, establece una serie de características de los CEE, tanto positivas como negativas: son una forma de inserción laboral; en muchos casos son ambientes acogedores, protectores y entre iguales, lo que proporciona sensación de seguridad; existe menos competitividad entre trabajadores; las familias

3 Lo que achaca, al menos en parte, a que la legislación no regula los procesos de transición. 
y los colectivos demandan este tipo de centros; se ven como lugares con menos ocasiones para la frustración personal, al contrario que las empresas ordinarias; para muchas personas con discapacidad son una meta, no un punto intermedio (independientemente de su tipo o grado de discapacidad); las personas con discapacidad tienen bajas perspectivas de acceso al mercado de trabajo ordinario; los trabajadores son más conformistas y están menos informados de sus derechos y obligaciones; existen escasas adaptaciones de los puestos de trabajo; hay una excesiva protección al trabajador que puede generar una falta de autonomía, y estos centros no tienen una imagen social construida, por lo que se pueden acabar convirtiendo en guetos. En definitiva, no hay una opinión clara sobre la idoneidad de estos centros como medio de inserción de las personas con discapacidad en el mercado de trabajo.

$\mathrm{Si}$, tal como se indicaba en el apartado anterior, los salarios de las personas con discapacidad son inferiores a los de los trabajadores sin discapacidad, parece existir consenso en que los salarios en los CEE son aún más bajos. Sin embargo, es prácticamente inexistente el análisis empírico al respecto. En este sentido, Rodríguez et alii (2012) analizan las diferencias salariales entre trabajadores con discapacidad en empresas ordinarias y CEE, y encuentran que más de la mitad de la diferencia salarial no se puede explicar por las diferentes características de ambos colectivos, aunque señalan que se debe ser cauto a la hora de atribuir estas diferencias a la discriminación, porque podemos encontrarnos ante diferencias en productividad que no podemos medir.

El Consejo Económico y Social (CES) (2003) destaca el crecimiento de los CEE, tanto en su número como en su plantilla, pero observa que se están apartando de su objetivo principal como puente al empleo ordinario, pues existe una alta tasa de temporalidad y se están integrando en su plantilla trabajadores con un grado de discapacidad medio-bajo. A pesar de esta crítica, el CES apoya a los CEE, siempre que mantengan su carácter social, así como su función de paso del empleo protegido al empleo ordinario, y se basen en criterios de viabilidad económica.

Finalmente, tal como se ha indicado anteriormente, otro aspecto que tener en cuenta a la hora de hablar del empleo protegido es su función como puente hacia el empleo ordinario. En general, aunque la evidencia empírica es escasa, hay consenso entre los autores sobre el escaso impacto que el empleo protegido tiene como forma de inserción en el mercado laboral ordinario (Pereda, 2003; López y Seco, 2005; Delsen, 2001; Visier, 1998). De Lorenzo (2004) destaca que el paso por un CEE es un buen punto de partida para adquirir experiencia laboral y comprobar las habilidades de la persona con discapacidad. Sin embargo, Malo 
y Rodríguez (2000) resaltan que los CEE suelen ser más bien el final de la carrera laboral de las personas con discapacidad que trabajan en ellos, y que existen incentivos en los CEE para que sus «mejores» trabajadores no sean promocionados al empleo ordinario, puesto que la productividad del CEE se vería afectada. Productividad que se espera que sea menor que la de un centro equivalente en el empleo ordinario, debido a que el CEE debe nutrirse solo con una parte del conjunto de la fuerza de trabajo (es decir, únicamente los trabajadores con discapacidad). Si los CEE no se encontraran protegidos en el mercado de bienes y servicios, su supervivencia como organización productiva se vería amenazada. Al estar protegidos del mercado abierto, es más probable que constituyan el fin de la carrera laboral de las personas con discapacidad (Malo y Muñoz-Bullón, 2006). Rodríguez (2012a) encuentra que los CEE no contribuyen a la inserción de las personas con discapacidad en el mercado de trabajo ordinario, sino que constituyen un destino en sí mismos. Aunque, tal como indica la autora, esto no implica que no favorezcan la inserción en el mercado de trabajo, es decir, el paso de este colectivo de la inactividad a la actividad.

En este sentido, no está claro que una obligación de un porcentaje mínimo de tránsito hacia la empresa ordinaria tuviera mejores resultados. Skedinger y Widerstedt (2006) encuentran que en Samhall (empresa sueca que proporciona empleo a personas con discapacidad y recibe subvenciones del Estado que dependen en parte de la consecución de ciertos objetivos como son priorizar la contratación de determinados grupos de personas con discapacidad y conseguir unos niveles de tránsito a la empresa ordinaria) la existencia de objetivos tanto sociales como económicos puede hacer que surjan conflictos. Esto puede hacer que se contrate a trabajadores sin discapacidad o que no estén dentro de los grupos priorizados, puesto que serán estos quienes de forma más probable lograrán encontrar otro empleo, o que se escoja a individuos con una discapacidad menos grave dentro de los grupos que priorizar (efecto que se suele denominar como cream skimming). Taylor (2003) coincide con estos autores al hacer referencia a la selección de los trabajadores más productivos, cuyo acceso al mercado de trabajo ordinario se «frena».

\section{La discapacidad y los CEE en la Muestra continua de vidas laborales}

En general, los análisis sobre discapacidad y mercado de trabajo en España utilizan bases de datos específicas para el estudio de la discapacidad que hacen referencia en algún apartado al mercado de trabajo (EDDES-1999, EDAD- 
2008), o bases genéricas sobre mercado de trabajo que incorporan información relativa a discapacidad (módulos especiales de la EPA, PHOGUE, ECV). ${ }^{4} \mathrm{La}$ fuente de información utilizada en este trabajo es la Muestra continua de vidas laborales (MCVL), que permite identificar a las personas que, al menos en un episodio de empleo, han presentado su certificado de discapacidad y también sus trayectorias laborales. Además, a partir del contrato específico para CEE, es posible identificar los CEE y así analizar las relaciones laborales en estos centros. Hasta el momento es la única base de datos que permite realizar este tipo de análisis. No se trata de una fuente específica para el estudio de la discapacidad, pero aporta información sobre las trayectorias laborales de los trabajadores en alta en la Seguridad Social en algún momento durante el año de referencia, constituyendo, por lo tanto, una de las pocas fuentes de datos que contienen esta información tan completa para España.

La principal diferencia entre la MCVL y el resto de bases de datos descritas en el párrafo anterior para el estudio de la discapacidad es que se trata de datos de registro administrativo, mientras que, en las otras, la situación de discapacidad se detecta a partir de distintas preguntas realizadas a las personas encuestadas. En la MCVL, la discapacidad se observa a partir de la información sobre el grado de discapacidad que el empresario declara al dar de alta al trabajador en una relación laboral, y para ello es necesario que el trabajador este en posesión del certificado de discapacidad y se lo comunique al empresario.

Las políticas activas dirigidas a personas con discapacidad en España están directamente relacionadas con la posesión del certificado de discapacidad, ya que este es el documento que acredita la discapacidad a efectos administrativos, y es necesario para ser beneficiario, entre otras, de las medidas de fomento del empleo (cuota de reserva, subvenciones, contratos específicos para personas con discapacidad, empleo selectivo o empleo protegido en CEE) o de las pensiones no contributivas por discapacidad. Por lo tanto, tiene sentido que, a la hora de estudiar el empleo en los $\mathrm{CEE},{ }^{5}$ identifiquemos a los trabajadores con discapacidad a partir del certificado de discapacidad.

Los datos sociodemográficos que nos facilita la MCVL son: sexo, año de nacimiento, provincia de nacimiento y de primera afiliación, nacionalidad, domicilio a nivel de municipio y fecha de fallecimiento. También proporciona información sobre la relación del individuo con la Seguridad Social como

4 Para más información sobre el análisis del empleo de las personas con discapacidad a partir de estas fuentes de información y de la MCVL, así como de los estudios realizados en España con ellas consultar Rodríguez (2013).

5 Para tener la condición de CEE, estos centros deben tener en su plantilla al menos un 70 por ciento de personas con discapacidad. Para más información sobre la regulación de estos centros consultar Cueto et alii (2008). 
cotizante. Para ello se tienen en cuenta las relaciones laborales de cada individuo, que no tienen por qué coincidir con sus contratos ni con situaciones de ocupación. ${ }^{6}$ La MVL facilita información sobre las características de los puestos de trabajo ocupados, tales como el grupo de cotización, el tipo de contrato, la fecha de inicio y de baja, la causa del cese y el tipo de actividad de la empresa. ${ }^{7}$

La MCVL nos permite estudiar las trayectorias laborales de los trabajadores seleccionados, lo que incluye episodios de empleo; episodios de percepción de prestaciones por desempleo, y percepción de pensiones contributivas, que pueden simultanearse con los episodios de empleo. Además, permite comparar las trayectorias laborales de los individuos antes y después de obtener el certificado de discapacidad (o al menos antes y después de presentarlo por primera vez). En cuanto al desempleo, esta base de datos no nos permite identificar los episodios de desempleo con exactitud, sino únicamente los episodios de percepción de prestación o subsidio por desempleo; no podemos identificar si los periodos en los que no aparece información sobre un trabajador se corresponden con desempleo no subsidiado o con inactividad. Sin embargo, nos permite diferenciar los periodos en los que los trabajadores perciben rendimientos del trabajo (independientemente de que sean salario o no), lo que les proporciona independencia económica, que es un pilar básico de la plena integración social de los individuos. Por lo tanto, se trata de una muestra representativa de la población trabajadora afiliada a la Seguridad Social de cada año, y proporciona toda la vida laboral de estos trabajadores. Están disponibles oleadas desde 2005 hasta 2011, por lo que es posible construir un panel de la situación laboral de cada trabajador con discapacidad en ese periodo.

La submuestra en la que se basa el estudio es el resultado de seleccionar a todas las personas que al menos en un episodio de empleo se identifican como «personas con discapacidad». Consideramos toda la trayectoria laboral de estas personas. Se han excluido episodios correspondientes a la percepción de las prestaciones por desempleo o las pensiones. Para identificar los CEE se ha utilizado una clave de tipo de contrato vigente, hasta el año 2001, que identifica los contratos realizados en este tipo de centros. A partir de esta clave se han localizado los códigos de cuenta de cotización de los centros para, posteriormente, identificar todos los contratos realizados en ellos. De esta manera podemos saber si las personas con discapacidad trabajan o no en un CEE, independientemente del

6 Cotizan a la Seguridad Social sin estar ocupados los beneficiarios de prestaciones o subsidios de desempleo así como aquellos que tienen un Convenio Especial de cotización.

7 Para más información consultar www.seg-social.es. 
tipo de contrato. En el estudio puede existir un sesgo a la baja ${ }^{8}$ en la medida en que hayan aparecido nuevos CEE a partir del año 2001, y también si tras 2001 alguien hubiera trabajado en un CEE que, simplemente por razones de muestreo, no hubiera aparecido en los momentos en que sí se podía identificar al empleador como un CEE. Sin embargo, esta base de datos es la única, hasta el momento, que nos permite realizar este tipo de análisis.

\section{Datos y análisis descriptivo}

La muestra final para el análisis empírico consiste en 9.006 individuos que comunicaron una discapacidad al menos en un episodio de empleo. Tenemos la trayectoria laboral de estos individuos antes y después de 2005. Esto implica que tenemos información sobre su experiencia previa, la duración del episodio de empleo seleccionado y los episodios posteriores de trabajo.

En el siguiente apartado se presentan las principales características de los trabajadores con contratos en alta en 2005 y las de dichos contratos.

\subsection{Características sociodemográficas}

En la tabla 3.1 se presentan las principales características de la submuestra seleccionada. Hay una mayor presencia de hombres $(66,8 \%)$, un resultado coincidente tanto con los observados en el mercado de trabajo español en general como dentro del colectivo de personas con discapacidad, en los que los hombres presentan una mayor tasa de empleo. Se observa una baja presencia de trabajadores mayores de 55 años, y el grueso de la muestra se encuentra entre los 30 y los 44 años de edad. También se puede observar la baja cualificación de los trabajadores que componen la muestra, siendo esta cualificación menor en el caso de los CEE. Hay una mayor proporción de trabajadores con un 33\% de discapacidad en las empresas ordinarias que en los CEE. Sin embargo, el porcentaje de trabajadores con un alto grado de discapacidad es similar en ambos tipos de empresas.

En cuanto al tipo de contrato, los contratos vigentes en 2005 son principalmente contratos indefinidos, aunque los contratos temporales representan el 39,6\% del total. Los episodios de empleo en CEE suponen el 8,9\% del total.

8 Sin embargo, Rodríguez (2012a) encuentra que el empleo en CEE sigue una distribución similar en la EDAD-2008 y la MCVL-2008, lo que parece indicar que el posible sesgo a la baja en la identificación de los CEE no es de gran magnitud. 
Tabla 4.1. Estadísticos descriptivos por tipo de empresa

\begin{tabular}{|c|c|c|c|c|c|c|}
\hline & \multicolumn{2}{|c|}{ CEE } & \multicolumn{2}{|c|}{ Empresa ordinaria } & \multicolumn{2}{|c|}{ Total } \\
\hline & $n$ & $\%$ & $n$ & $\%$ & $n$ & $\%$ \\
\hline Hombres: & 477 & 59,4 & 5.536 & 67,5 & 6.013 & 66,8 \\
\hline \multicolumn{7}{|c|}{ Edad (2005): } \\
\hline$<30$ & 125 & 15,6 & 1.704 & 20,8 & 1.829 & 20,3 \\
\hline $30-44$ & 387 & 48,2 & 3.889 & 47,4 & 4.276 & 47,5 \\
\hline $45-54$ & 223 & 27,8 & 1.856 & 22,6 & 2.079 & 23,1 \\
\hline$>54$ & 68 & 8,5 & 754 & 9,2 & 822 & 9,1 \\
\hline \multicolumn{7}{|c|}{ Grado de discapacidad: } \\
\hline $33 \%$ & 237 & 29,5 & 3.190 & 38,9 & 3.427 & 38,1 \\
\hline $34-64 \%$ & 422 & 52,6 & 3.482 & 42,4 & 3.904 & 43,3 \\
\hline$>64 \%$ & 144 & 17,9 & 1.531 & 18,7 & 1.675 & 18,6 \\
\hline \multicolumn{7}{|c|}{ Cualificación: } \\
\hline Alta & 14 & 1,7 & 457 & 5,6 & 471 & 5,2 \\
\hline Media & 376 & 46,8 & 5.403 & 65,9 & 5.779 & 64,2 \\
\hline Baja & 413 & 51,4 & 2.343 & 28,6 & 2.756 & 30,6 \\
\hline
\end{tabular}

Fuente: Cálculos de los autores con la MCVL

\subsection{Contratos vigentes en 2005}

Las características de los episodios de empleo en función del tipo de empresa se muestran en la tabla 4.2. Los episodios de empleo en CEE se distribuyen en mayor medida entre empresas de gran tamaño que en el caso de las empresas ordinarias. Casi el $50 \%$ de los episodios de empleo en las empresas ordinarias se han celebrado con empresas de menos de once empleados, mientras que esta dimensión representa menos del 20\% de los episodios de empleo en CEE. Por el contrario, las empresas de más de 50 empleados representan casi dos de cada tres contratos en CEE, pero menos del $20 \%$ en empresas ordinarias.

Con respecto a la distribución de los episodios por sector, hay algunas diferencias interesantes entre CEE y empresas ordinarias. Los episodios en CEE se encuadran principalmente en la industria, la sanidad y los servicios a empresas, mientras que, en las empresas ordinarias, la concentración de episodios en estos sectores es menor. Aunque los tres sectores citados son también los que tienen los porcentajes más altos de episodios, los porcentajes son más bajos. Además, el comercio y la construcción también emplean aproximadamente al 10\% de los trabajadores. 
Tabla 4.2. Estadísticos descriptivos por tipo de empresa. Características de los episodios de empleo

\begin{tabular}{|c|c|c|c|c|c|c|}
\hline & \multicolumn{2}{|c|}{ CEE } & \multicolumn{2}{|c|}{ Empresa ordinaria } & \multicolumn{2}{|c|}{ Total } \\
\hline & $n$ & $\%$ & $n$ & $\%$ & $n$ & $\%$ \\
\hline \multicolumn{7}{|l|}{ Tipo de contrato: } \\
\hline Indefinido & 540 & 67,2 & 4.840 & 59,0 & 5.380 & 59,7 \\
\hline Temporal & 263 & 32,8 & 3.289 & 40,2 & 3.562 & 39,6 \\
\hline \multicolumn{7}{|c|}{ Edad al inicio del episodio: } \\
\hline$<30$ & 244 & 30,4 & 2.387 & 29,1 & 2.631 & 29,2 \\
\hline $30-44$ & 369 & 46,0 & 3.809 & 46,4 & 4.178 & 46,4 \\
\hline $45-54$ & 153 & 19,1 & 1.559 & 19,0 & 1.712 & 19,0 \\
\hline$>54$ & 37 & 4,6 & 448 & 5,5 & 485 & 5,4 \\
\hline \multicolumn{7}{|c|}{ Año de inicio del episodio: } \\
\hline$<1990$ & 20 & 2,4 & 146 & 1,9 & 176 & 2,0 \\
\hline 1991-1995 & 59 & 7,3 & 185 & 2,3 & 244 & 2,7 \\
\hline 1996-2000 & 186 & 23,2 & 842 & 10,3 & 1.028 & 11,4 \\
\hline 2001-2004 & 416 & 51,8 & 5.126 & 62,5 & 5.542 & 61,5 \\
\hline 2005 & 122 & 15,2 & 1.894 & 23,1 & 2.016 & 22,4 \\
\hline \multicolumn{7}{|c|}{ Tamaño de la empresa (no trabajadores) } \\
\hline$<10$ & 158 & 19,7 & 3.928 & 47,9 & 4.086 & 45,4 \\
\hline $11-20$ & 31 & 3,9 & 548 & 6,7 & 579 & 6,4 \\
\hline $21-50$ & 105 & 13,1 & 773 & 9,4 & 878 & 9,7 \\
\hline $51-250$ & 260 & 32,4 & 1.371 & 16,7 & 1.631 & 18,1 \\
\hline$>250$ & 249 & 31,0 & 1.583 & 19,3 & 1.832 & 20,3 \\
\hline \multicolumn{7}{|l|}{ Sector: } \\
\hline Agricultura & 10 & 1,2 & 106 & 1,3 & 116 & 1,3 \\
\hline Industria & 214 & 26,7 & 1.262 & 15,4 & 1.476 & 16,4 \\
\hline Construcción & 8 & 1,0 & 858 & 10,5 & 866 & 9,6 \\
\hline Comercio & 21 & 2,6 & 1.144 & 13,9 & 1.165 & 12,9 \\
\hline Hostelería & 7 & 0,9 & 452 & 5,5 & 459 & 5,1 \\
\hline Transportes & 13 & 1,6 & 285 & 3,5 & 298 & 3,3 \\
\hline Sanidad & 180 & 22,4 & 1.417 & 17,3 & 1.597 & 17,7 \\
\hline Educación & 21 & 2,6 & 152 & 1,9 & 173 & 1,9 \\
\hline Servicios financieros & 5 & 0,6 & 117 & 1,4 & 122 & 1,4 \\
\hline Servicios a empresas & 240 & 29,9 & 1.392 & 17,0 & 1.632 & 18,1 \\
\hline Otros servicios & 84 & 10,5 & 593 & 7,2 & 677 & 7,5 \\
\hline
\end{tabular}

Fuente: Cálculos de los autores con la MCVL 
Dado que el objetivo de este trabajo es estudiar la estabilidad en el empleo, prestaremos especial atención a los contratos indefinidos, puesto que los contratos temporales son, por definición, más inestables. Centrándonos únicamente en estos contratos, tabla 4.3, observamos resultados muy similares a los del total de la muestra. La principal diferencia es el menor peso del sector de la construcción en el caso de las empresas ordinarias.

Tabla 4.3. Estadísticos descriptivos por tipo de empresa. Contratos indefinidos

\begin{tabular}{|c|c|c|c|c|c|c|}
\hline & \multicolumn{2}{|c|}{ CEE } & \multicolumn{2}{|c|}{ Empresa ordinaria } & \multicolumn{2}{|c|}{ Total } \\
\hline & $n$ & $\%$ & $n$ & $\%$ & $n$ & $\%$ \\
\hline \multicolumn{7}{|c|}{ Edad al inicio del episodio: } \\
\hline$<30$ & 188 & 34,8 & 1.309 & 26,9 & 1.497 & 27,6 \\
\hline $30-44$ & 253 & 46,9 & 2.296 & 47,1 & 2.549 & 47,1 \\
\hline $45-54$ & 82 & 15,2 & 981 & 20,1 & 1.063 & 19,6 \\
\hline$>54$ & 17 & 3,1 & 289 & 5,9 & 306 & 5,7 \\
\hline \multicolumn{7}{|c|}{ Año de inicio del episodio: } \\
\hline$<1990$ & 20 & 3,7 & 150 & 3,1 & 170 & 3,1 \\
\hline 1991-1995 & 56 & 10,4 & 176 & 3,6 & 232 & 4,3 \\
\hline 1996-2000 & 179 & 33,1 & 797 & 16,3 & 976 & 18,0 \\
\hline 2001-2004 & 269 & 49,8 & 3367 & 69,1 & 3636 & 67,1 \\
\hline 2005 & 16 & 3,0 & 385 & 7,9 & 401 & 7,4 \\
\hline \multicolumn{7}{|c|}{ Tamaño de la empresa ( $n^{\circ}$ trabajadores) } \\
\hline$<10$ & 111 & 20,6 & 2.242 & 46,0 & 2.353 & 43,5 \\
\hline $11-20$ & 18 & 3,3 & 353 & 7,2 & 371 & 6,9 \\
\hline $21-50$ & 73 & 13,5 & 452 & 9,3 & 525 & 9,7 \\
\hline $51-250$ & 191 & 35,4 & 776 & 15,9 & 967 & 17,9 \\
\hline$>250$ & 147 & 27,2 & 1.052 & 21,6 & 1.199 & 22,1 \\
\hline \multicolumn{7}{|l|}{ Sector: } \\
\hline Agricultura & 9 & 1,7 & 71 & 1,5 & 80 & 1,5 \\
\hline Industria & 137 & 25,4 & 930 & 19,1 & 1.067 & 19,7 \\
\hline Construcción & 5 & 0,9 & 322 & 6,6 & 327 & 6,0 \\
\hline Comercio & 17 & 3,1 & 800 & 16,4 & 817 & 15,1 \\
\hline Hostelería & 4 & 0,7 & 258 & 5,3 & 262 & 4,8 \\
\hline Transportes & 7 & 1,3 & 172 & 3,5 & 179 & 3,3 \\
\hline Sanitario & 118 & 21,9 & 1.060 & 21,7 & 1.178 & 21,8 \\
\hline Educación & 17 & 3,1 & 71 & 1,5 & 88 & 1,6 \\
\hline Servicios financieros & 4 & 0,7 & 77 & 1,6 & 81 & 1,9 \\
\hline Servicios a empresas & 153 & 28,3 & 662 & 13,6 & 815 & 1,5 \\
\hline Otros servicios & 69 & 12,8 & 347 & 7,1 & 416 & 15,1 \\
\hline
\end{tabular}

Fuente: Cálculos de los autores con la MCVL 
En la tabla 4.4 se presentan algunos datos sobre la trayectoria laboral de los trabajadores con discapacidad. Al igual que en las tablas anteriores, dividimos la muestra de trabajadores con discapacidad en dos grupos: los que en 2005 están trabajando en CEE y los que lo hacen en empresas ordinarias. Los trabajadores de la muestra han comenzado a trabajar en torno a los 22 años (en el caso de los trabajadores en CEE la edad es ligeramente superior). La experiencia laboral previa al episodio de empleo vigente en 2005 es de 9,5 años en el caso de trabajadores en empresas ordinarias y 8,4 años en el caso de trabajadores de CEE. La proporción de trabajadores con experiencia en el desempleo es menor entre las personas que trabajan en CEE. Sin embargo, los que han estado en esta situación han experimentado periodos de desempleo más largos que los trabajadores de empresas ordinarias. Por último, la relación anterior de los trabajadores con los CEE es superior entre aquellos que trabajan en estos centros en 2005. Tal como se indicaba en el apartado anterior, se trata de un resultado esperado porque los CEE son un mercado de trabajo protegido para las personas con discapacidad, que puede acabar convirtiéndose en el punto final de su carrera.

Las cifras correspondientes a los trabajadores con contratos indefinidos son similares a las de la muestra. Las diferencias entre los trabajadores en empresas ordinarias y en CEE son similares.

Tabla 4.4. Trayectorias laborales anteriores al episodio de empleo de 2005.

\begin{tabular}{|l|c|c|c|c|}
\hline & \multicolumn{2}{|c|}{ Total } & \multicolumn{2}{c|}{ Contr. Indefinidos } \\
\hline & $\begin{array}{c}\text { Empresas } \\
\text { ordinarias }\end{array}$ & CEE & $\begin{array}{c}\text { Empresas } \\
\text { ordinarias }\end{array}$ & CEE \\
\hline Antigüedad en el episodio de empleo & 4,8 & 7,3 & 7,3 & 9,8 \\
\hline Edad en el primer episodio & 21,7 & 22,9 & 20,8 & 21,2 \\
\hline Tiempo trabajado & 9,5 & 8,4 & 12,0 & 11,1 \\
\hline Algún episodio de desempleo & $58,6 \%$ & $48,6 \%$ & $56,3 \%$ & $39,6 \%$ \\
\hline Tiempo en desempleo & 1,9 & 2,1 & 1,8 & 2,0 \\
\hline Alguna relación con CEE & $10,9 \%$ & $62,6 \%$ & $11,1 \%$ & $66,7 \%$ \\
\hline
\end{tabular}

Fuente: Cálculos de los autores con la MCVL

\subsection{Permanencia en el empleo}

A partir del panel elaborado podemos analizar si los contratos existentes en 2005 siguen vigentes en los años sucesivos hasta 2011 (ver figura 4.1) y si los individuos se mantienen en el empleo independientemente de si es en el mismo episodio o no. La proporción de contratos aún en vigor en 2011 es del 29,6\% en el 
caso de CEE y del 17,9\% en el de las empresas ordinarias. Si nos centramos en el porcentaje de trabajadores que está fuera de la muestra en 2011, este es del 41,2\% en el caso de trabajadores en CEE y del $47 \%$ para los trabajadores de empresas ordinarias. Este resultado se puede poner en relación con la crisis económica

Los trabajadores que en 2011 no están en el mismo episodio de empleo que en 2005 se encuentran principalmente en el empleo ordinario, independientemente del tipo de empresa en la que prestaran servicios en 2001. Esta situación se da en mayor medida entre los trabajadores que en 2005 prestaban servicios en una empresa ordinaria: un $95,2 \%$ de los que tienen un empleo diferente en 2011 se mantienen en este tipo de centros. En cuanto al tránsito desde el CEE a la empresa ordinaria, un 60,7\% de los individuos que en 2005 prestaban servicios en un CEE y que en 2011 están en un episodio diferente están en empresas ordinarias.

Figura 4.1. Transiciones desde los contratos vigentes en 2005 en función del tipo de empresa.

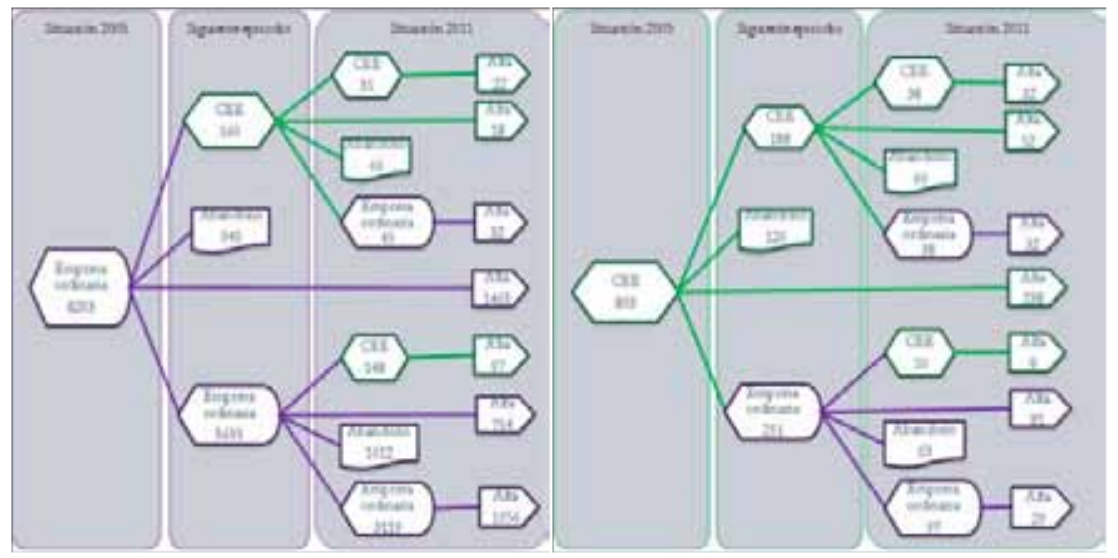

Fuente: Cálculos de los autores con la MCVL

Tal como se indicaba anteriormente, dado que el objetivo de este trabajo es estudiar la estabilidad en el empleo, a continuación repetimos el análisis de transiciones únicamente con los contratos indefinidos en 2005 (figura 4.2). La proporción de contratos indefinidos que siguen en situación de alta en 2011 es del $39,8 \%$ en el caso de los CEE y del 29,2\% en el de las empresas ordinarias. Por lo tanto. se observa que la estabilidad sigue siendo mayor en los CEE, aunque se reducen ligeramente las diferencias con las empresas ordinarias. En cuanto al porcentaje de trabajadores que han abandonado el mercado laboral, en este caso las diferencias entre CEE y empresas ordinarias se incrementan; han abandona- 
do el mercado de trabajo un 29,8\% de los trabajadores de CEE y un 38,4\% de los de empresas ordinarias.

En cuanto al tránsito a la empresa ordinaria, un 32,2\% de los trabajadores que en 2005 tenían un contrato indefinido en un CEE y que han cambiado de episodio están en 2011 en una empresa ordinaria (casi la mitad que para el total de la muestra; resulta lógico que los contratos temporales tengan una mayor relación con el tránsito a la empresa ordinaria que los indefinidos). En este caso se observa que, entre los trabajadores que han cambiado de episodio de empleo, únicamente un $50,3 \%$ de los trabajadores con contrato indefinido en una empresa ordinaria en 2005 se mantienen en la empresa ordinaria, mientras que prácticamente en la misma proporción están en CEE. Este resultado puede estar relacionado con la labor de los CEE como punto de retorno (empleo refugio) de trabajadores con discapacidad cuando no se pueden mantener en la empresa ordinaria (por ejemplo, por problemas de adaptación debido a un empeoramiento de su discapacidad).

Figura 4.2. Transiciones desde los contratos indefinidos vigentes en 2005 en función del tipo de empresa.
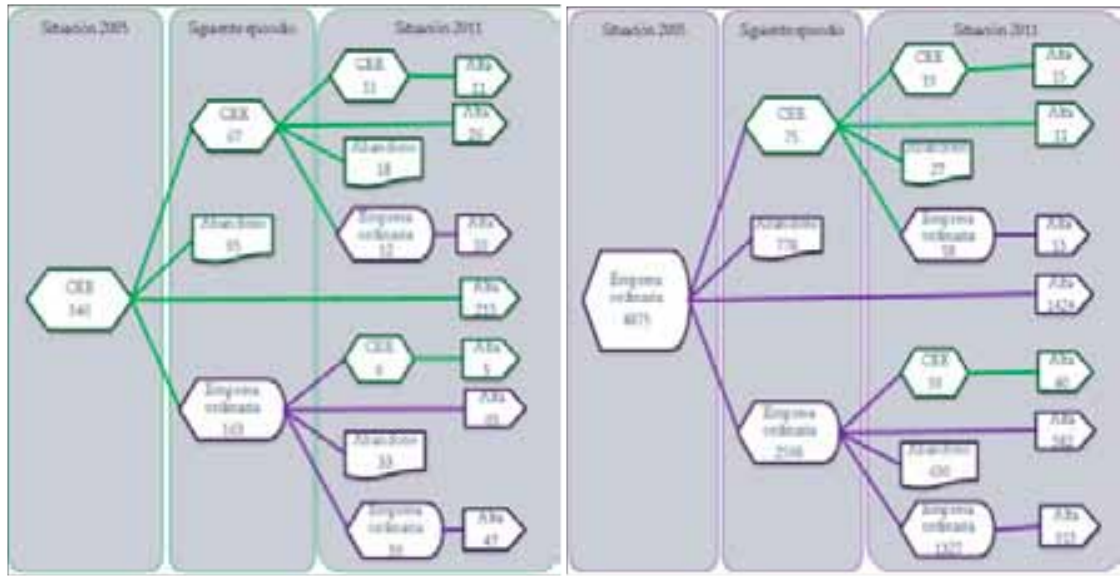

Fuente: Cálculos de los autores con la MCVL

Por lo tanto, los CEE parecen ofrecer puestos de trabajo más estables que las empresas ordinarias. Sin embargo, es difícil para los trabajadores en los CEE encontrar un trabajo en una empresa ordinaria. Cuando pierden sus puestos de trabajo, por lo general, pasan a trabajar en otros CEE o abandonan mercado de trabajo. Por otro lado, aunque prácticamente la totalidad de los trabajadores que en 2005 prestaban servicios en una empresa ordinaria y que han cambiado de empleo se mantienen en la empresa ordinaria, si nos centramos únicamente en 
los contratos indefinidos estos resultados se reducen prácticamente a la mitad Podemos relacionar esta situación con la labor de los CEE como empleo refugio, que «recoge» a los trabajadores con discapacidad que son expulsados del mercado de trabajo ordinario. Estos resultados están en la línea de los obtenidos por Rodríguez (2012b).

\section{Conclusiones}

Los CEE constituyen la principal política activa del mercado laboral dirigida a los trabajadores con discapacidad en España. Los objetivos de estos centros son la reducción del desempleo y de la inactividad de los trabajadores con discapacidad, así como el fomento de su integración en el mercado laboral ordinario. La evidencia empírica no es clara acerca del cumplimiento de estos objetivos. El trabajo en estos centros ofrece una carrera más estable, con una movilidad inferior a la experimentada al trabajar en empresas ordinarias. Sin embargo, los empleados suelen tener situaciones profesionales menos favorables (salarios más bajos y peores condiciones de trabajo), por lo que el empleo en los CEE puede conducir a la inmovilidad y la inestabilidad de los trabajadores con discapacidad.

El objetivo de este trabajo era estudiar si el empleo en los CEE es más estable que el empleo en empresas ordinarias utilizando un panel de datos elaborado con la MCVL a partir de las oleadas de 2005 hasta 2011. Nuestros resultados confirman que los trabajadores con discapacidad en CEE tienen empleos más estables que los trabajadores con discapacidad en empresas ordinarias. Las personas con discapacidad que trabajan en los CEE en 2005 tienen una mayor probabilidad de mantenerse en el mismo empleo después de seis años que aquellos contratados por empresas ordinarias.

Además, diversos aspectos de las carreras profesionales de los trabajadores en CEE parecen indicar una mayor estabilidad de sus relaciones laborales, lo que, en principio, se podría interpretar como una mejor situación laboral respecto a aquellos que no prestan servicios en un CEE; los trabajadores de estos centros experimentan una menor rotación y, además, los CEE parecen ser un «refugio» para personas con discapacidad de baja cualificación. Sin embargo, es probable que tras estas situaciones se esconda una mayor precariedad de este colectivo: una menor rotación puede significar estancamiento en unas condiciones laborales no satisfactorias, y la baja cualificación de los trabajadores en CEE puede estar escondiendo una infracualificación de su personal, que tiene como efecto directo un salario inferior al percibido por las personas con discapacidad en empresas ordinarias. 
Sin embargo, una pregunta abierta es si son programas públicos dirigidos a CEE los que les permiten ofrecer más estabilidad a sus trabajadores. Teniendo en cuenta las políticas de apoyo y las subvenciones que estas empresas reciben cuando contratan a trabajadores con discapacidad, podríamos pensar que este apoyo contribuye a la estabilidad de estos trabajadores. Sin embargo, si, como indica gran parte de la literatura, las condiciones de trabajo en los CEE no son tan buenas como en empresas ordinarias, podríamos estar en una situación en que las ayudas públicas estén favoreciendo el estancamiento en unas condiciones laborales precarias. Cabe plantearse si unas peores condiciones de trabajo con una mayor estabilidad son preferibles a unas buenas condiciones de trabajo con menor estabilidad. Esta cuestión abre una importante línea de investigación con respecto a la integración laboral y social de personas con discapacidad.

Los datos disponibles no permiten el análisis de los CEE como un medio para aumentar la participación de los trabajadores con discapacidad en el mercado laboral. Sin embargo, es posible estudiar su papel en la prevención de que estos trabajadores abandonen el mercado de trabajo una vez que se emplean. Nuestros resultados refuerzan la imagen de CEE como un método para mantener activos a los trabajadores con discapacidad y hacerles participar en el mercado laboral. Sin embargo, estos resultados deben ser considerados en relación con los recursos públicos que reciben estos centros respecto a las empresas ordinarias, dado que la estabilidad del empleo en CEE puede estar asociada con la financiación pública que reciben o con otros aspectos. En consecuencia, es necesaria más investigación para disponer de información suficiente con respecto a estas cuestiones, y para ello es deseable la realización de un análisis coste-beneficio del empleo de las personas con discapacidad.

\section{Referencias bibliográficas}

Albor, J. (1997): El empleo y el trabajo protegido CEE. Seminario Internacional Empleo y personas con retraso mental, Cáceres, 1997.

BALdwin, M. y Johnson, W.G. (1995): «Labor market discrimination against women with disabilities». Industrial Relations, 34(4), pp. 555-577.

- (2000): «Labor market discrimination against men with disabilities in the year of the ADA». Southern Economic Journal, 66(3), pp. 548-566.

Baldwin, M. y Schumacher, E.J. (2000): «Wages, Quality Sorting, and the Job Characteristics of Workers with Disabilities». Working Paper Ecu 17. Department of Economics, East Carolina University. Recuperado el 5 de mayo, de: <http://www.ecu.edu/cs-educ/econ/upload/ecu0017.pdf> 
Brown, C.L. y Emery, J.C.H. (2008)ः «The Impact of Disability on Earnings and Labour Force Participation in Canada: Evidence from the 2001 PALS». Working Paper 2008-26. Department of Economics, University of Calgary. Recuperado el 5 de mayo, de: <http://econ.ucalgary.ca/sites/econ.ucalgary. ca/files/publications/PALSworkingpaper2008.pdf>

Casado, D. (2004): «Conocimiento y gestión del empleo de las personas con discapacidad». Revista del Ministerio de Trabajo y Asuntos Sociales, 50, pp. 47-72.

Consejo Económico y Social (2003): La situación de las personas con discapacidad en España, en Informes 2003, Consejo Económico y Social, Madrid.

Comisión Europea (2000): Benchmarking employment policies for people with disabilities. ECOTEC Research and Consulting Ltd.

Cueto, B.; Malo, M.A.; Rodríguez, V.y Francos, C. (2008): Trayectorias laborales de las personas con discapacidad y Centros especiales de empleo: análisis empirico con la Muestra de Vidas Laborales. Recuperado el 5 de mayo, de: <http:// www.seg-social.es/prdi00/groups/public/documents/binario/119779.pdf>

Cueto, B.; Malo, M.A. y Rodríguez, V. (2012): La brecha de participación laboral de las personas con discapacidad en España. Un análisis con la EDAD2008. Cuadernos del Mercado de Trabajo, 8, pp. 20-27.

DÁvila, C.D. (2003)ः «Perfil educativo y participación laboral de los jóvenes con discapacidad». Comunicación presentada en las XII Jornadas de la Asociación de Economía de la Educación. Getafe, 18 y 19 septiembre 2003. Recuperado el 5 de mayo, de: <http://www.economicsofeducation.com/wpcontent/ uploads/oviedo2005/MT09.pdf>

- (2006): «Discapacidad y género. Un estudio de participación en el mercado de trabajo español». Moneda y Crédito, 223, pp. 127-158.

- y Malo, M.A. (2006): "Género, discapacidad y posición familiar: la participación laboral de las mujeres con discapacidad». Cuadernos Aragoneses de Economía, 16(1), pp. 61-82.

Delsen, L. (1996): «Employment opportunities for the disabled». En Schmid, G.; O’Really, J. y Schöman, K. (eds.), International Handbook of Labour Market Policy and Evaluation, capítulo 17,pp.520-550. Cheltenham: Edward Elgar.

- (2001): Economic study of special employment of disabled people in the European Union. Paper EG0123 University of Nijmegen.

Gannon, B. y Nolan, B. (2004): «Disability and labour market participation». Equality Studies Unit Report. Recuperado el 5 de mayo, de: <http://www. equality.ie/Files/Disability\%20and\%20Labour\%20Market\%20Participation. pdf $>$ 
Gill, M. (2005): «The myth of transition: contractualizing disability in the sheltered workshop», Disability E Society, 20(6), pp. 613-623.

Giménez, D. y Ramos, M. (2003): «La discriminación de las mujeres discapacitadas en España», Revista del Ministerio de Trabajo y Asuntos Sociales, 45, pp. 61-76.

Humer, B.; Wuellrich, J. P. \& Z Zeimüller, J. (2007): «Integrating severely disabled individuals into the Labour Market: the Austrian case». IZA Discussion Paper, Núm. 2649. Recuperado el 5 de mayo, de: <http://ftp.iza. org/dp2649.pdf>

Ibáñez, P. y Mudarra, Ma J. (2005): «Relaciones sociales en el empleo, en trabajadores con discapacidad». Educación XXI: Revista de la Facultad de Educación, 8, pp. 105-125.

Johnson, W.y LAMBrinos, J. (1985): «Wage discrimination against handicapped men and women», Journal of Human Resources, 20(2), pp. 264-277.

Jones, M.; Latreille, P. y Sloane, P. (2006): «Disability, gender, and the British labour market». Oxford Economic Papers, 58(3), pp. 407-449.

López Pino, C. y Seco Martín, E. (2005): «Discapacidad y empleo en España: su visibilidad». Innovar Revista de Ciencias Administrativas y Sociales, 15 (26), pp. 59-72.

Lorenzo, R. de (2004)ः «El futuro de los discapacitados en el mundo: el empleo como factor determinante para la inclusión». Revista del Ministerio de Trabajo y Asuntos Sociales, 50; pp. 73-89.

Malo, M.A.y Rodríguez, S. (2000)ः «Centros especiales de empleo: una breve reflexión económica sobre los cambios recientes de su normativa». Documentación Laboral, 61, pp. 181-193.

- (2001): «Discapacidad y participación en el mercado de trabajo». En GarRIdo, Ly Toharia, L. Condiciones de vida en España y en Europa, pp. 281-306. Madrid: Instituto Nacional de Estadística.

- (2003): «Las personas con discapacidad en el mercado de trabajo español». Revista del Ministerio de Trabajo y Asuntos Sociales, 46; pp. 99-126.

- (2004): «¿Cómo afectan las discapacidades a la probabilidad de ser activo en España? Un análisis empírico con datos de la Encuesta sobre Discapacidades, Deficiencias y Estado de la Salud de 1999». Cuadernos de Economía, 27, pp. 75-108.

- y Muñoz-Bullón, F. (2006): Employment promotion measures and the quality of the job match for persons with disabilities. Hacienda Pública Española / Revista de Economía Pública, 179, pp. 79-111. 
-, Cueto, B.; Dávila, D;; Pagán, R. y Rodríguez, V. (2009): Informe de Evaluación sobre la politica de bonificación de cuotas para las personas con discapacidad en situación de desempleo. Agencia de Evaluación y Calidad. Recuperado el 5 de mayo, de: http //www.aeval.es/comun/pdf/evaluaciones/E19-2009.pdf

- y PAGÁN, R. (2012): «Wage differentials across Europe: discrimination and/ or lower productivity?» International Labor Review, 151(1-2), pp. 43-60.

O'Reilly, A. (2007): The right to decent work of persons with disabilities. ILO.

Organización para la Cooperación y el Desarrollo Económicos (1992): Employment policies for people with disabilities-Report by an evaluation panel. OECD Labour Market and Social Policy Occasional Papers Núm. 8.

- (2003): Transforming disability into ability: Policies to Promote Work and Income Security for Disabled People. Paris: Servicio de Publicaciones de la OCDE.

- (2007): New ways of addressing partial work capacity. Recuperado el 5 de mayo, de: <http://www.oecd.org/social/socialpoliciesanddata/38509814.pdf>

Pagán, R. y Marchante, A. (2004): «Diferencias salariales por discapacidad en España: el caso de los varones». Hacienda Pública Española/Revista de Economía Pública, 171(4); pp. 75-100.

Pagán, R. y Malo, M.A (2009), «Job satisfaction and disability: Lower expectations about jobs or a matter of health». Spanish Economic Review, 11(1), pp. 51-74.

PAGÁN, R. (2011a): «Ageing and disability: Job satisfaction differentials across Europe». Social Science E Medicine, 72 (2), pp. 206-215.

- (2011b): «Self-employment and job satisfaction: Evidence for older people with disabilities in Europe». European Journal of Ageing, 8 (3), pp. 177-187.

- (2012): «Transitions to part-time work at older ages: The case of people with disabilities in Europe». Disability E Society, 27 (1), pp. 95-115.

Pascual, M. y Cantarero, D. (2007): «Socio-demographic determinants of disabled people: An empirical approach based on the European Community Household Panel».Journal of Socio-Economics, 36(2), pp. 275-287.

Pereda, C.; de Prada, M.A. y Actis, W. (2003): La inserción laboral de las personas con discapacidades. Colección Estudios Sociales Fundación La Caixa Núm. 14.

Rodríguez, V. (2012a): Discapacidad y mercado de trabajo: Tres análisis empiricos con la Muestra Continua de Vidas Laborales. Estudios de la Fundación, Serie Tesis. Madrid: FUNCAS.

Rodríguez, V. (2012b): «El empleo de las personas con discapacidad en la gran recesión: ¿Son los Centros Especiales de Empleo una excepción?» Estudios de Economía Aplicada, 30(1), pp. 237-259. 
- (2013): «Fuentes de información sobre discapacidad y empleo en España». Revista Española de Discapacidad, 1(1). Próxima publicación.

- Malo, M.A. y Cueto, B. (2012): «Diferencias salariales por discapacidad y Centros Especiales de Empleo». Cuadernos de Economía, 35(98) pp. 100-116. Rubio, F.J. (2003): «La construcción social del empleo protegido: los centros especiales de empleo». Nómadas. Revista Crítica de Ciencias Social y Jurídicas, 8. Shima, I.; Zólyomi, E. y Zaidi, A. (2008): The Labour Market Situation of People with Disabilities in EU25. Policy Brief, 1, European Centre.

Skedinger, P. y Widerstedt, B. (2007): «Cream skimming in employment programmes for the disabled? Evidence from Sweden». International Journal of Manpower, 28(8), pp. 694-714.

TAYLOR, S. J. (2003) Disabled workers deserve real choices, real jobs. Recuperado el 5 de mayo, de: <www.accessiblesociety.org/topics/economics-employment/ shelteredwksps.html>

Thornton, P. y Lunt, N. (1997): Employment Policies for Disabled People in Eighteen Countries: A Review. Gladnet Collection. Cornell University. Recuperado el 5 de mayo, de: <http://digitalcommons.ilr.cornell.edu/cgi/ viewcontent. .cgi?article $=1158 \&$ context $=$ gladnetcollect $>$

Villa, N. (2003): «Situación laboral de las personas con discapacidad en España». Revista Complutense de Educación. 14(2), pp.393-424.

Visier, L. (1998): «Relaciones laborales en los sistemas de trabajo protegido para personas minusválidas». Revista internacional del Trabajo, 117(3), pp. 371-390.

Whiteford, P. (2007): The active social policy agenda - an OECD perspective. Inclusion in working life ISSA - European Regional Meeting Oslo 1516/05/2007. Recuperado el 5 de mayo, de: <http://www.issa.int/esl/ Ressourcen/Tagungsberichte/Die-Agenda-zur-aktiven-Sozialpolitik-einePerspektive-der-OECD $>$.

Zwinkels, W. (2001). «The employment situation of people whit disabilities in the European Union». Research paper, EIM Business and Policy Research. Recuperado el 5 de junio, de: <http://www.socialdialogue.net/docs/si_key/ Employment_Disabled2_en.pdf> 